\title{
Retrospective analysis of the clinical features of outpatients with novel coronavirus in areas outside Wuhan, China
}

\section{Yong-Tao Li}

1 State Key Laboratory for Diagnosis and Treatment of Infectious Diseases, National Clinical Research Center for Infectious Diseases, Collaborative Innovation Center for Diagnosis and Treatment of Infectious Diseases, Department of Infectious Diseases, The First Affiliated Hospital, College of Medicine, Zhejiang University

\section{Mei-Lian Peng}

2 Zhejiang Provincial People's Hospital, Hangzhou

\section{Jia-dan Fu}

1 State Key Laboratory for Diagnosis and Treatment of Infectious Diseases, National Clinical Research Center for Infectious Diseases, Collaborative Innovation Center for Diagnosis and Treatment of Infectious Diseases, Department of Infectious Diseases, The First Affiliated Hospital, College of Medicine, Zhejiang University

\section{Li Liu}

1 State Key Laboratory for Diagnosis and Treatment of Infectious Diseases, National Clinical Research Center for Infectious Diseases, Collaborative Innovation Center for Diagnosis and Treatment of Infectious Diseases, Department of Infectious Diseases, The First Affiliated Hospital, College of Medicine, Zhejiang University

\section{Yong-zheng Guo}

1 State Key Laboratory for Diagnosis and Treatment of Infectious Diseases, National Clinical Research Center for Infectious Diseases, Collaborative Innovation Center for Diagnosis and Treatment of Infectious Diseases, Department of Infectious Diseases, The First Affiliated Hospital, College of Medicine, Zhejiang University

\section{Jian-Rong Huang}

1 State Key Laboratory for Diagnosis and Treatment of Infectious Diseases, National Clinical Research Center for Infectious Diseases, Collaborative Innovation Center for Diagnosis and Treatment of Infectious Diseases, Department of Infectious Diseases, The First Affiliated Hospital, College of Medicine, Zhejiang University

\section{Ji-Fang Sheng}

1 State Key Laboratory for Diagnosis and Treatment of Infectious Diseases, National Clinical Research Center for Infectious Diseases, Collaborative Innovation Center for Diagnosis and Treatment of Infectious Diseases, Department of Infectious Diseases, The First Affiliated Hospital, College of Medicine, Zhejiang University 


\section{Lan-Juan Li}

1 State Key Laboratory for Diagnosis and Treatment of Infectious Diseases, National Clinical Research Center for Infectious Diseases, Collaborative Innovation Center for Diagnosis and Treatment of Infectious Diseases, Department of Infectious Diseases, The First Affiliated Hospital, College of Medicine, Zhejiang University

\section{Kai-jin Xu ( $\sim$ zdyxyxkj@zju.edu.cn )}

1 State Key Laboratory for Diagnosis and Treatment of Infectious Diseases, National Clinical Research Center for Infectious Diseases, Collaborative Innovation Center for Diagnosis and Treatment of Infectious Diseases, Department of Infectious Diseases, The First Affiliated Hospital, College of Medicine, Zhejiang University

\section{Research Article}

Keywords: novel coronavirus pneumonia, myocardial zymogram, novel coronavirus, fever

Posted Date: April 28th, 2020

DOI: https://doi.org/10.21203/rs.3.rs-25843/v1

License: (c) (i) This work is licensed under a Creative Commons Attribution 4.0 International License. Read Full License 


\section{Abstract}

Objective: The novel coronavirus pneumonia (COVID-19) has spread rapidly across the globe with the movement of people. How to diagnose COVID-19 quickly and accurately is a concern for all. We retrospectively assessed the clinical characteristics of patients with COVID-19 detected by outpatient screening in areas outside Wuhan, China, to guide early screening outside the epidemic area, to isolate and treat COVID-19-positive patients, and to control the spread of this virus in the region.

Results: Among the 213 patients treated in the fever clinic of our hospital, 41 tested positive for novel coronavirus (2019-nCoV) and 172 were negative. Among the positive patients, 13 (31.7\%) of the patients had been to Wuhan, while 28 (68.3\%) had not been to Wuhan. There were 4 cases of clustering occurrence. The main symptoms exhibited by COVID-19-positive patients were fever (87.8\%), cough (68.3\%), and expectoration (34.1\%). The C-reactive protein (CRP) levels were increased in 35 (85.3\%) positive patients; the hydroxybutyrate dehydrogenase in the myocardial zymogram was increased in 22 positive patients $(53.6 \%)$ and 38 negative patients (22.1\%); computed tomography (CT) findings revealed lung lesions in all 41 positive patients (100\%).

Conclusion: We classified the patient population and analyzed the data to understand the early clinical performance of COVID-19. Our research illustrate that screening for COVID-19 outside Wuhan should focus on early symptoms such as fever and cough, in combination with lung CT findings, epidemiological history, and sputum pathogen detection to determine whether patients need further isolation.

\section{Introduction}

In December 2019, a novel acute respiratory disease emerged in the Wuhan Province. This infectious disease is caused by the 2019-nCoV, and it rapidly spread to other regions. ${ }^{[1]}$ Since then, there have been case reports from 24 countries across 5 continents, which has caused great concern worldwide. ${ }^{[2-6]}$ The 2019-nCoV infectious pneumonia (NCIP) has spread rapidly across the globe with the movement of people. To block the source of infection and to control the spread of the virus, it is particularly important to screen for people who are infected with the 2019-nCoV outside its place of origin. In our clinical work, we found that some of those positive for the 2019-nCoV had been to Wuhan; others had not, but have had local contact with people returning from Wuhan; some of the affected patients have not been to Wuhan, and have not come in contact with people who had been to Wuhan; we even found positive patients with no apparent epidemiological history and positive patients with no obvious symptoms. Therefore, clarifying the clinical characteristics of NCIP outpatients outside Wuhan can further improve the screening accuracy and achieve early detection, early diagnosis, early isolation, and early treatment.

\section{Research Design}

This was a retrospective study that was approved by the Ethics Committee of the First Affiliated Hospital of Zhejiang University School of Medicine and met institutional and national guidelines, and all of the 
patients were informed of the retrospective review and signed informed consent forms before the clinical data were collected. All the patients who attended the fever clinic at the First Affiliated Hospital of Zhejiang University from January 22 to February 8, 2020, were included in the study. After the patient provided oral consent, he or she was included in the retrospective study. The final follow-up date was February 22, 2020. Scope of the screening: We divided the scope of the screening into four categories: 1) people who returned from Wuhan or Hubei within 14 days; 2) those who were in contact with patients who had been diagnosed in this area within 14 days; 3) those who had been in contact with recent returnees from Wuhan within 14 days; 4 ) those with no obvious epidemiological history as above but exhibiting recent symptoms of cough, fever, throat discomfort, chest tightness, and other respiratory symptoms. Exclusion criteria included: 1) Patients with two negative sputum tests for 2019-nCoV RNA taken at an interval of 24 hours, and with no obvious abnormalities on lung CT scan were considered as negative for 2019-nCoV infection. These patients were excluded from the study, and prescribed home isolation for 2 weeks with a follow-up thereafter. 2) Patients with two negative sputum tests but presenting with infectious lesions in the lung CT scan; however, their CT images were not similar to those of confirmed patients. These patients were provided treatment in a single room in the general ward and followed up thereafter. 3) Patients with two negative sputum tests and presenting with infectious lesions in the lung CT scan, and the images were similar to lung CT images in confirmed patients. These patients were provided symptomatic treatment in single room isolation, and the sputum test was repeated.

\section{Data collection}

We analyzed the medical records of patients who went to the fever clinic. Epidemiological, clinical, laboratory, and radiological characteristics, as well as treatment and outcome data were all obtained from data collection forms in electronic medical records. The recorded information included demographic data, medical history, exposure history, potential comorbidities, symptoms, signs, laboratory results, and chest CT scan. The onset date was defined as the date on which the symptoms appeared. Real-time reverse transcription polymerase chain reaction was carried out to detect $\mathrm{nCoV}$ according to the recommendation of the National Institute for Viral Disease Prevention and Control (China) (http://ivdc.chinacdc.cn/kyjz/202001/t20200121_211337. html). Sputum samples were collected, and the total RNA was extracted within 2 hours of sample collection using a respiratory sample RNA isolation kit, and real-time reverse transcription polymerase chain reaction (RT-PCR) was used to detect 2019-nCoV RNA. ${ }^{[7]}$

\section{Statistical analysis}

Categorical variables were described in terms of frequency and percentage, and continuous variables were described as means \pm standard deviation or median and interquartile range (IQR) values. For normally distributed data, an independent $t$ test was used to compare the mean of continuous variables; otherwise, the Mann-Whitney test was used. Proportions of categorical variables were compared using 
the $X^{2}$ test. All statistical analyses were performed using SPSS 19.0 software. A p value of $<0.05$ were considered statistically significant.

\section{Patient and public involvement}

This was a retrospective case series study and no patients were involved in the study design, setting the research questions, or the outcome measures directly. No patients were asked to advise on interpretation or writing up of results.

\section{Results}

\section{Clinical}

Of the 213 patients, there were 94 men and 119 women. The average age was 44.3 years. Among the 213 patients, 41 were positive for $2019-n C o V$ and 172 were negative. The commonly encountered symptoms in these 41 patients included fever (36 cases, 87.8\%), cough (28 cases, 68.3\%), expectoration (14 cases, $34.1 \%$ ), muscle soreness (10 cases, $24.4 \%$ ), fatigue (9 cases, $22.0 \%)$, chest tightness ( 8 cases, $19.5 \%)$, headache ( 6 cases, $14.6 \%$ ), chills ( 6 cases, 14.6 ), and anorexia ( 3 cases, $7.3 \%)$. Among them, 1 positive patient was asymptomatic, but her sputum was tested as her husband had a positive diagnosis, and it tested positive for 2019-nCoV RNA, and her lung CT results presented with obvious lesions. The main symptoms of the 172 negative patients were fever (122 cases, 70.93\%), cough (104 cases, 60.5\%), expectoration (42 cases, $24.1 \%$ ), and sore throat (44 cases, $25.6 \%$ ). Among positive patients, fever symptoms were statistically significant compared with negative patients. Among all the 213 outpatients, the vital signs were stable, and the heart rate, blood pressure, and respiratory rate were within the normal range. (Tables 1 and 2 ).

\section{Epidemiological}

Of the 213 outpatients, 50 had been to Wuhan. Of these 50,13 tested positive for 2019 -nCoV while the remaining 37 tested negative. A total of 18 patients had contact with confirmed patients, of which 10 were positive and 8 were negative. and 54 patients had contact with returnees from Wuhan, of which 8 were positive and 46 were negative; 91 patients had no clear epidemiological history, of which 10 were positive and 81 were negative. There was statistical significance between patients with and without contact history. Of the 41 positive patients, $13(31.7 \%)$ had been to Wuhan; $28(68.3 \%)$ had not been to Wuhan. Of the 172 negative patients, $37(21.5 \%)$ had been to Wuhan, $8(4.7 \%)$ had been contacted with confirmed patients,,46 (26.7\%) had been contacted with recent returnees from Wuhan, and $81(47.1 \%)$ had no clear contact history (Table 1 ).

Of the 41 positive patients (26.8\%), there were 12 clustering occurrences, and there were 4 cases of familial clustering: one was a couple (the husband was in contact with returnees from Wuhan), one had 6 
cases (colleagues in the same unit who had contact with returnees from Wuhan), and the other two were a couple and a father and son, neither of whom had a clear contact history. The average time from onset to diagnosis of 41 positive patients was 5.1 days; this was 4.6 days among patients who had been to Wuhan $(n=13)$ and 5.3 days for patients who had not been to Wuhan. There was no statistically significant difference between the two (Table 2).

\section{Underlying disease}

Of the 41 positive patients, 7 had underlying diseases: 5 had hypertension, 1 had diabetes, and 1 had chronic kidney disease.

\section{Inspection result}

1. General laboratory tests: All the patients underwent routine blood test, liver and kidney function tests, myocardial zymogram, erythrocyte sedimentation rate (ESR), and Compared with 2019-nCoVnegative patients, 2019-nCoV-positive patients had significantly lower levels of peripheral blood leukocytes, neutrophils, and lymphocytes. The CRP levels were significantly higher in 2019-nCoVpositive patients (35 cases, $85.3 \%$ ) than in 2019 -nCoV-negative patients ( 75 cases, $3.6 \%$ ). In the myocardial zymogram, 2019-nCoV-positive patients showed significantly higher enzyme levels than 2019-nCoV-negative patients: lactate dehydrogenase was elevated in 13 2019-nCoV-positive patients (31.7\%) and 14 2019-nCoV-negative patients (8.1\%); hydroxybutyrate dehydrogenase was increased in 22 positive patients (53.6\%) and 38 negative patients (22.1\%), creatine kinase (CK) was increased in 1 positive patient and 0 negative patients, and creatine kinase-MB (CK-MB) was elevated in 1 positive patient and 1 negative patient. The ESR level was increased in 22 positive cases (53.6\%) and in 64 negative cases (37.2\%), and there was no significant difference between the two. In terms of liver function, the alanine aminotransferase (ALT) levels were elevated in 4 positive cases $(9.7 \%$ ) and 7 negative cases $(4.1 \%)(p<0.05)$, the aspartate aminotransferase (AST) levels were increased in 5 positive cases $(12.1 \%)$ and 10 negative cases $(5.8 \%)(p<0.05)$; total bilirubin was increased in 2 positive cases $(4.8 \%)$ and 3 negative cases $(1.7 \%)(p>0.05)$. In terms of renal function, creatinine levels were elevated in 5 of the positive cases $(12.1 \%)$ and 4 of the negative cases $(2.3 \%)(p<0.05)$; urea nitrogen was elevated in 3 positive patients $(7.3 \%)$ and 6 negative patients (3.4\%); uric acid was increased in 1 positive case $(2.4 \%)$ and 8 negative cases $(4.6 \%)(p>0.05)$. We found that there were no statistically significant differences in all the laboratory tests between positive patients who had been to Wuhan and those who had not. (Tables 3 and 4).

2. Etiological examination: Of the 41 positive patients, 38 (92.7\%) tested positive in the first sputum test, $1(2.4 \%)$ tested positive in the second sputum test, $1(2.4 \%)$ tested positive in the sixth sputum test, and another patient $(2.4 \%)$ tested positive in the fifth sputum

3. Imaging and pulmonary function tests: Lung CT scans were performed in all the screened Lung CT showed lesions indicative of pneumonia in 41 cases (100\%), and lesions were also observed in 69 
negative cases $(40.1 \%)$, and there was a statistically significant difference between the two groups. Lung lesions in positive cases were primarily local patchy shadows or ground-glass shadows in one lung or bilaterally (Figures 1 and 2). Negative cases showed various lung lesions, which were commonly observed as sheet density shadow (Figure 3).

\section{Diagnosis time}

The average time from the onset to the diagnosis of pneumonia among the 41 positive patients was 5.1 days (range, 1-12 days). The average times of diagnosis among patients who had been to Wuhan and those who had not were 4.6 and 5.3 days, respectively. There was no statistically significant difference between the two. A total of 38 cases were diagnosed in the first sputum test, 1 case was diagnosed in the second sputum test carried out 24 hours later. Two patients were very special. One tested negative in the second sputum test despite having a history of contact with returnees from Wuhan, and collectively contacted colleagues from the unit that had clustering occurrence. Lung CT for this patient revealed suspected lesions (Figure 1), single-room isolation screening was continued, and the sixth sputum test was positive. The second patient had a negative result in the second sputum test but the lung CT revealed suspected lesions (Figure 2), single-room isolation was continued and 2 additional sputum tests remained negative. As he did not have a clear history of contact with people from Wuhan or with a confirmed patient, he was transferred to a single room in the general ward, and the fifth sputum test was positive. Among the 173 negative patients, 103 had no obvious abnormalities in the lung $C T$, and the second sputum test was negative, and they were excluded. There were another 69 cases with lesions on lung $\mathrm{CT}$, the lesions were revealed to be atypical (Figure 3). The second sputum test of these patients was negative, and considering their epidemiological history, they were considered free of infection.

\section{Discussion}

We retrospectively analyzed patients with 2019-nCoV infection in our hospital, which is located outside Wuhan. The clinical manifestations of patients with COVID-19 infection were mainly fever, cough, expectoration, muscle soreness, fatigue, chest tightness, and headache, which were similar to those observed among 2019-nCoV patients in Wuhan. ${ }^{[7]}$ These symptoms were also similar to the common upper respiratory tract or lung infections in patients who tested negative for 2019-nCoV. Some 2019-nCoV patients showed gastrointestinal symptoms such as bloating, anorexia, and diarrhea. One patient had no symptoms, but had a clear history of contact with positive patients. This patient had a mild lung infection, but it required special attention as a special infection source. Other literatures have also reported such patients. ${ }^{[8]}$ Fever is the most important symptom of the 2019-nCoV infection, which occurs in more than $87 \%$ of patients, which is higher than patients who tested negative in the 2019 -nCoV screening during the same period. Fever has also been reported in literature as the most important symptom of patients with COVID-19, exceeding $80 \%$. ${ }^{\text {[9] }}$ 
Results of the laboratory tests revealed that the leukocyte, neutrophil, and lymphocyte counts in 2019nCoV-positive patients was significantly than that of negative patients. More than $85 \%$ of the infected patients had elevated CRP levels, which was more meaningful than the CRP levels in negative patients, indicating that the inflammatory response was a more common phenomenon in 2019-nCoV-positive patients. ${ }^{[10]}$ A small number of 2019-nCoV-positive patients in our study had mild liver dysfunction, which was statistically significant compared to 2019-nCoV-negative patients, indicating that 2019-nCoV could cause mild liver dysfunction. A small number of positive patients also had abnormal renal function, reflected by increased creatinine, urea, and uric acid levels. Compared with the negative patients in the same time period, the positive patients exhibited a significant increase in the creatinine level, indicating that the 2019-nCoV can also cause early renal damage; this needs our attention. More than $53 \%$ of the 2019-nCoV positive patients in our study had abnormal myocardial zymogram, which was mainly manifested by elevated hydroxybutyrate dehydrogenase, and this difference was statistically significant compared with that in negative patients. We compared and analyzed the clinical symptoms and laboratory indicators of 2019-nCoV-infected patients who had been to Wuhan and those who had not, and found no significant difference between the two groups, indicating that there were no significant differences in early symptoms and laboratory tests of infected people in different regions.

We found that patients with positive 2019-nCoV had lung CT lesions, and only $40 \%$ of 2019 -nCoVnegative patients had abnormal lung CT. There were also reports of early patients showing no signs of pneumonia on the chest radiographs on day 4 of symptom onset. ${ }^{[11]}$ The time from onset to diagnosis of our patients was about 5 days. We believed that lung CT was a more sensitive approach and could detect lung lesions earlier.

In our study, we also found an asymptomatic case. The patient was married to 1 patient with confirmed infection. After the husband was diagnosed, the wife was tested although she did not exhibit any clinical symptoms. She had a positive sputum test and a CT scan of her lungs showed infection. We thought that early-stage patients with COVID-19 have no clinical symptoms, but exhibited a small range of lesions in lung CT. Therefore, early detection using lung CT scanning has positive significance for patients due to an early diagnosis. Additionally, we found one patient who was negative for multiple sputum tests but had an epidemiological history. Lung images in this patient revealed lesions that were consistent with the virus infection, isolation screening was continued and the sixth sputum test was positive. Some scholars also performed bronchoscopic bronchoalveolar lavage fluid on viral RNA for inpatients to further confirm the diagnosis. ${ }^{[1]}$ We also found that one patient had obvious lesions on lung CT, but this patient had no obvious history of contact. After the second sputum test was negative, CT images of the lungs were considered to be more in line with viral infection lesions, and two sputum tests were negative. After being transferred to the general ward for 1 day, the fifth sputum test was positive. Therefore, we believe that the presence of lesions in the lung CT should be the main basis for initiating clinical treatment, and a positive sputum test is the main basis for isolating patients and controlling the source of infection. For patients with negative secondary sputum tests, if there are obvious lesions on the lung CT suggesting viral infection, sputum screening needs to be continued. 
Epidemiological history is usually one of the main parameters used to judge infectious diseases.

Our data showed that 13 of the 50 patients who had been to Wuhan were positive; 10 of 18 patients who had been in contact with confirmed patients were positive; 8 of 54 people who had been contact with returnees from Wuhan were positive. For areas outside the epidemic area, when considering the humanto-human susceptibility, a second or even third generation of infected persons will appear. Therefore, there is also a risk of infection for those who have no epidemiological history. ${ }^{[12]}$ We also found that of the 91 patients with no clear epidemiological history, 10 were infected with 2019-nCoV. We also found four cases of clustering occurrence. In one case, 20 employees from the same unit had a meeting with one person from Wuhan, and 6 of the 20 people acquired the infection. In one case with fever and cough, lung CT scan showed an obvious infection, and the sixth sputum test was positive. The remaining three cases were a familial clustering occurrence: two couples, a father and son, further clarifying that 2019$\mathrm{nCoV}$ can be transmitted from person to person. ${ }^{[12]}$

In this study, the average time from the onset of symptoms to the diagnosis of 41 positive patients was approximately 5 days. Among these patients, the average time from symptom onset to diagnosis of those who returned from Wuhan was 4.6 days, and that of those who had not been to Wuhan was 5.3 days; however, there were no statistically significant differences between the two. Considering that many of the people experiencing fever, cough, and other symptoms had not been to Wuhan, most did not expect that they would be infected with the 2019-nCoV. These subjects thought that they were suffering from a common upper respiratory infection and did not go to the hospital clinic in time for targeted screening. In fact, according to our data, of the 41 positive patients who were screened at the outpatient clinic of the hospital, 38 (92.7\%) were positive for the first sputum test, i.e., they were confirmed positive on the day of consultation. One case (2.4\%) was positive for the second sputum test, i.e., the diagnosis was confirmed on the second day of consultation; only one case was confirmed on the $12^{\text {th }}$ day. Therefore, we should increase public awareness and understanding of the disease in order to encourage hospital visits for early diagnosis, early isolation, and early treatment.

Our research revealed that screening for the 2019-nCoV outside Wuhan is an urgent task. The results of our retrospective analysis revealed that our four-point criteria for the exclusion of 2019-nCoV is significant: it is vital to accurately identify positive patients among many patients with fever, cough, or other symptoms, or even among asymptomatic patients with no obvious epidemiological history, so as to avoid missed diagnosis and to control the infection source at an early stage. Of course, our results will inevitably be biased due to the relationship between time and number of cases.

\section{Declarations}

Funding

No funding.

Declaration of Competing Interest 
The authors declare that they have no competing interests

\section{References}

1. Zhu N, Zhang D, Wang W, et al. A novel coronavirus from patients with pneumonia in China, NEngl J Med 2020; 382: 727-733.DOI: 10.1056/NEJMoa2001017.

2. Novel coronavirus (2019-nCoV): Situation report-5, 25 January 2020. Geneva: World Health Organization,

3. Xu XW, Wu XX, Jiang XG, et al.Clinical findings in a group of patients infected with the 2019 novel coronavirus (SARS-Cov-2) outside of Wuhan, China: retrospective case series. BMJ 2020: 368, m792. DOI: 1136/bmj.m792.

4. Chan JF-W, Kok K-H, Zhu Z, et al. Genomic characterization of the 2019 novel human-pathogenic coronavirus isolated from a patient with atypical pneumonia after visiting Emerging Microbes \& Infections 2020;9:221-36. DOI: 10.1080/22221751.2020.1719902.

5. Gralinski LE, Menachery Return of the Coronavirus: 2019-nCoV. Viruses 2020;12:135. DOI: 10.3390/v12020135.

6. Paraskevis D, Kostaki EG, Magiorkinis G,et al. Full-genome evolutionary analysis of the novel corona virus (2019-nCoV) rejects the hypothesis of emergence as a result of a recent recombination event. Infect Genet Evol 2020;79:104212. DOI: 1016/j.meegid.2020.104212.

7. Wang D, Hu B, Hu C,et al. Clinical Characteristics of 138 Hospitalized Patients With 2019 Novel Coronavirus-Infected Pneumonia in Wuhan, China. JAMA 2020;Feb 7[Online ahead of print]. DOI: 1001/jama.2020.1585.

8. Rothe C, Schunk M, Sothmann P,et al. Transmission of 2019-nCoV Infection From an Asymptomatic Contact in $N$ Engl J Med2020;Jan:30[Online ahead of print]. DOI: 10.1056/NEJMc2001468.

9. Chen NS, Zhou M, Dong X,et al. Epidemiological andclinical characteristics of 99 cases of 2019 novel coronavirus pneumonia inWuhan, China: a descriptive Lancet 2020; 395:507-513. DOI: 10.1016/S0140-6736(20)30211-7.

10. Zhang JN, Zhou LQ, Yang YQ, et al. Therapeutic and Triage Strategies for 2019 Novel Coronavirus Disease in Fever Clinics. Lancet Respir Med 2020; Feb 13[Online ahead of print]. DOI: 1016/S22132600(20)30071-0

11. Holshue ML, DeBolt C, Lindquist S, et al. First Case of 2019 NovelCoronavirus in the United States. N Engl J Med 2020; Jan 31[Online ahead of print]. DOI: 1056/NEJMoa2001191.

12. Phan LT, Nguyen TV, Luong QC, et al. Importation and Human-to-Human Transmission of a Novel Coronavirus in Vietnam. N Engl J Med 2020; 382:872-874. DOI: 1056/NEJMc2001272.

\section{Tables}

Due to technical limitations, the tables are only available as a download in the supplemental files section. 


\section{Figures}
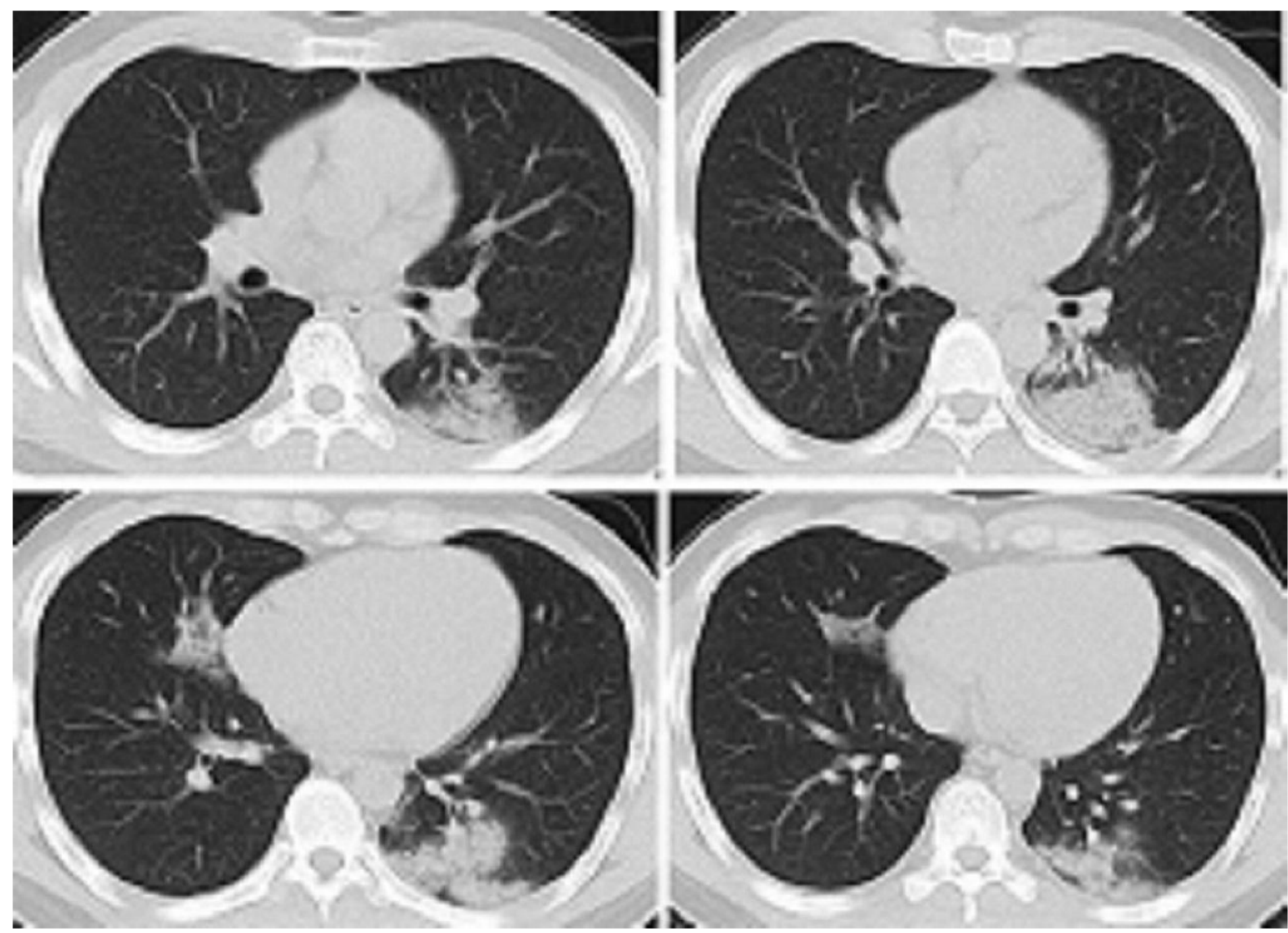

\section{Figure 1}

Lung CT of patients diagnosed with 6 sputum tests: patchy blurry density shadows in the right middle lobe and lower left lung. Lesions were distributed around the lungs, and locally showed ground-glass density shadows. 

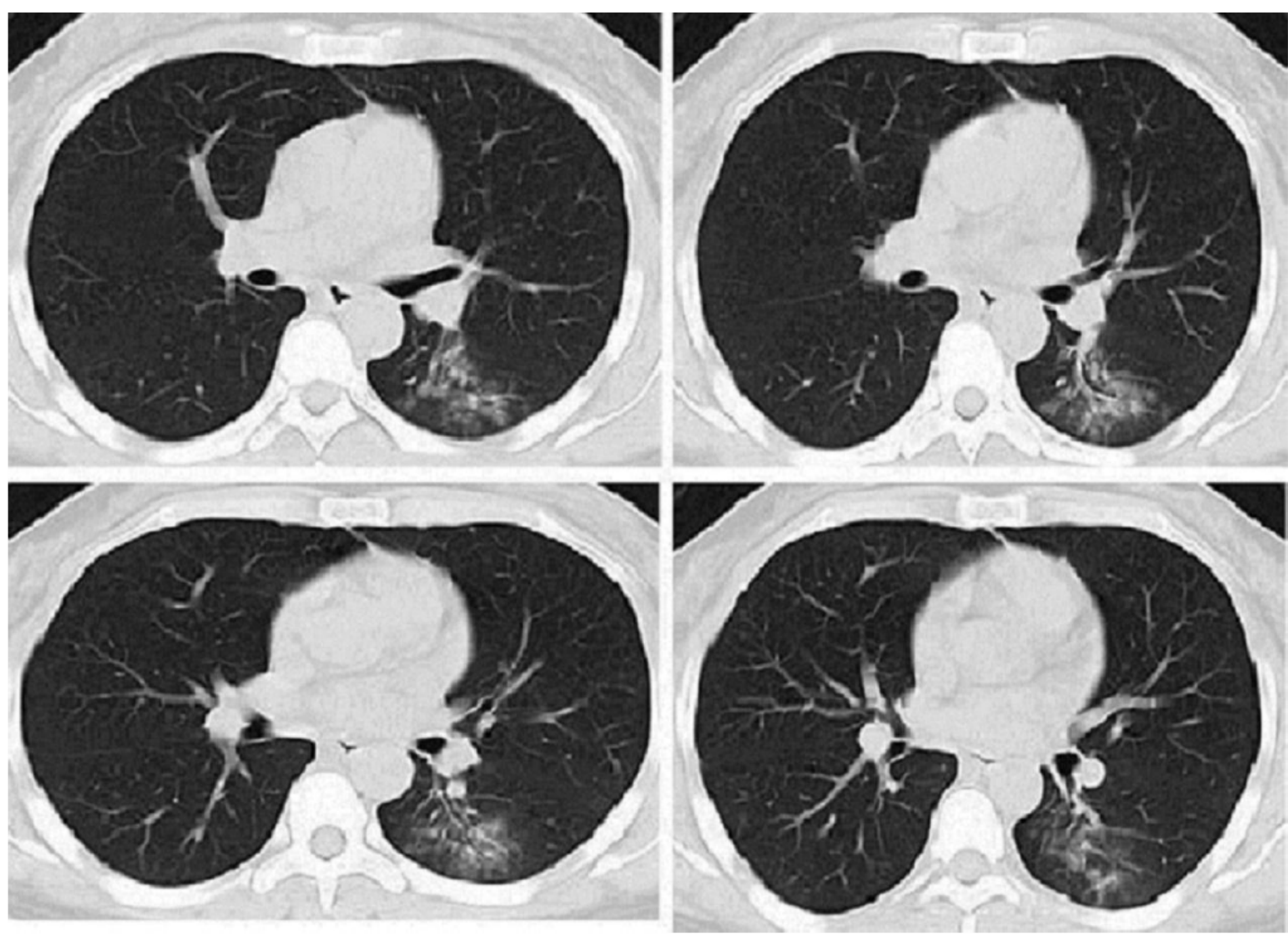

Figure 2

Lung CT of patients with four negative and positive sputum tests after transferring to general ward: the dorsal segment of the lower left lung lobe was nodular, with patchy blurry high-density shadows. 

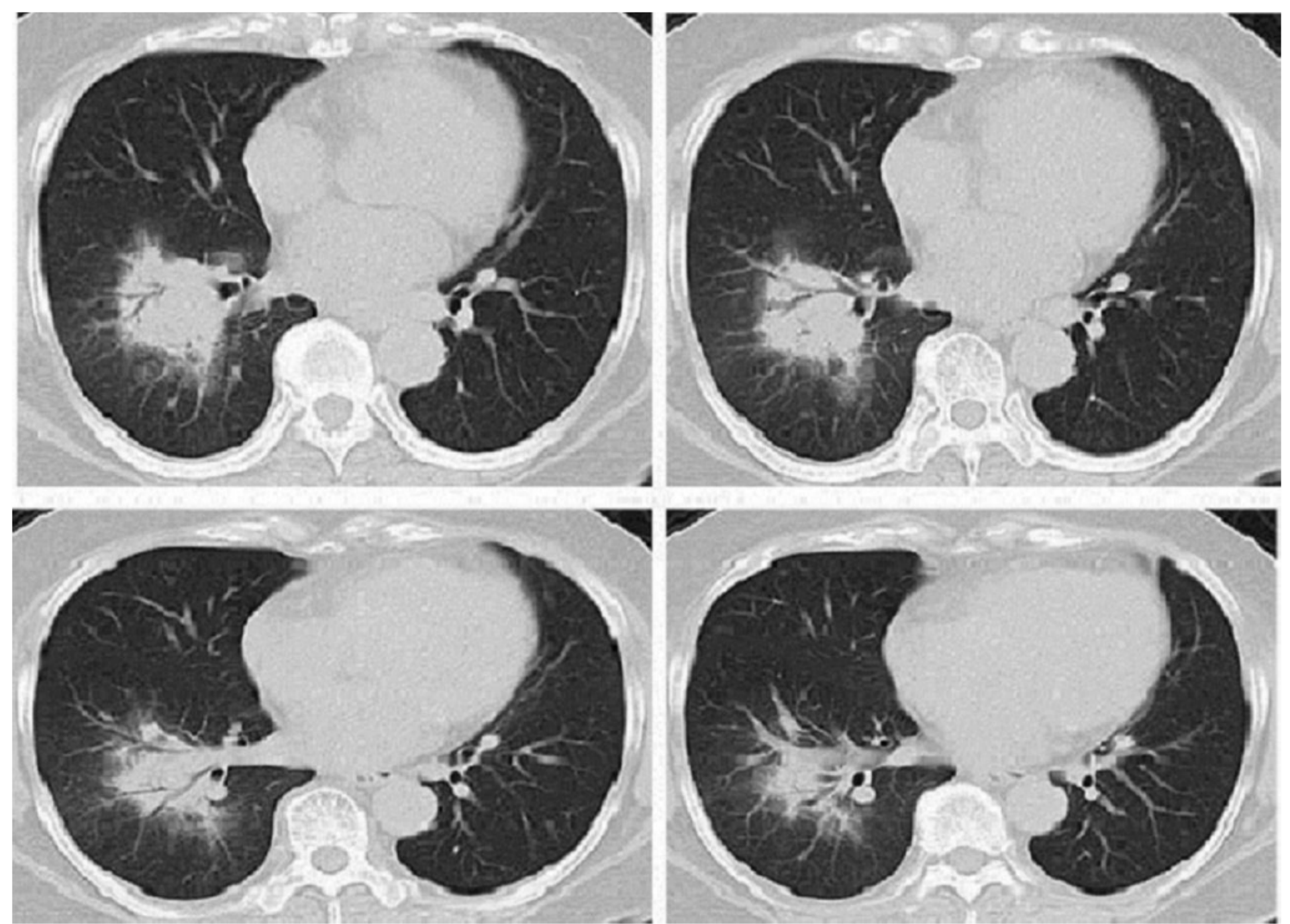

\section{Figure 3}

Lung CT of patients with common pulmonary infection with a negative second sputum test: parahilar sheet density shadows in the lower right lung lobe.

\section{Supplementary Files}

This is a list of supplementary files associated with this preprint. Click to download.

- Tables.pdf 